\title{
Apport De La Télédétection Et De La Géophysique Dans La Cartographie Des Fractures Hydrauliquement Actives En Zone De Socle Au Centre-Ouest Du Benin
}

\section{Akokponhoué Yanonvoh Nesny, Msc}

Chaire Internationale en Physique Mathématique et Applications (CIPMA Chaire UNESCO), Université d'Abomey-Calavi, Cotonou, République du Benin. Laboratoire d'Hydrologie Appliqué, Institut National de l'Eau (INE),

Université d'Abomey-Calavi, Cotonou, République du Benin

Yalo Nicaise, $M C$

Laboratoire d'Hydrologie Appliqué, Institut National de l'Eau (INE),

Université d'Abomey-Calavi,Cotonou, République du Benin

\section{Akokponhoué Houngnigbo Bertrand, PhD}

Laboratoire d'Hydrologie Appliqué, Institut National de l'Eau (INE),

Université d'Abomey-Calavi, Cotonou, République du Benin

\section{Youan Ta Marc, MC}

Département des Sciences et Techniques de l'Eau et du Génie de l'Environnement, U.F.R des Sciences de la Terre et des Ressources Minières

(STRM), Université de Cocody, Côte d'Ivoire

\section{Agbahoungba George, PhD}

Chaire Internationale en Physique Mathématique et Applications

(CIPMA Chaire UNESCO), Université d'Abomey-Calavi, Cotonou,

République du Benin

Doi:10.19044/esj.2019.v15n27p426 URL:http://dx.doi.org/10.19044/esj.2019.v15n27p426

\section{Résumé}

La région des collines est célèbre au Bénin pour ses problèmes de ravitaillement en eau car située dans une zone constituée uniquement de roches cristallines. Elle connait des difficultés aiguës d'accessibilité aux ressources en eau. La présente étude a pour objectifs de cartographier les réseaux de fractures issues des images satellitaires et d'utiliser la tomographie de résistivité électrique pour leurs localisations au sol. L'approche méthodologique utilisée est basée sur l'utilisation des techniques de la télédétection pour la cartographie structurale des linéaments et l'application de la tomographie de résistivité électrique pour la validation des linéaments hydrauliquement actives. L'utilisation des techniques de l'Analyse en 
Composantes Principales Sélectives et des filtrages spatiaux directionnels de Sobel (7x7) ont permis d'établir la carte des linéaments majeurs. La validation des linéaments a été faite au cours de plusieurs travaux de prospection géophysique de tomographie de résistivité électrique. La carte générée à la suite du traitement des images satellitaires compte 638 structures linéaires dont la taille varie de 7 à $34 \mathrm{~km}$ et qui présentent deux directions préférentielles $\mathrm{N} 0^{\circ}-\mathrm{N} 10^{\circ}$ et $\mathrm{N} 80^{\circ}-\mathrm{N} 90^{\circ}$. A l'issu des travaux de géophysique, 13 linéaments ont été validés en fractures majeures avec des largeurs de la zone fissurée-altérée allant de 15 à $40 \mathrm{~m}$ et dont leurs profondeurs sont au-delà de $50 \mathrm{~m}$. La gamme de résistivité électrique caractérisant ces fractures varie de 100 à $800(\Omega . m)$.

Mots clés: Linéaments, Tomographie de résistivité électrique, Roches Cristallines, Ressources en eau, Bénin 


\title{
Contribution of Remote Sensing and Geophysics in the Mapping of Hydraulically Active Fractures in Hard Rock in West Central Benin
}

\begin{abstract}
Akokponhoué Yanonvoh Nesny, Msc
Chaire Internationale en Physique Mathématique et Applications (CIPMA Chaire UNESCO), Université d'Abomey-Calavi, Cotonou, République du Benin. Laboratoire d'Hydrologie Appliqué, Institut National de l'Eau (INE), Université d'Abomey-Calavi, Cotonou, République du Benin
\end{abstract}

Yalo Nicaise, $M C$

Laboratoire d'Hydrologie Appliqué, Institut National de l'Eau (INE),

Université d'Abomey-Calavi,Cotonou, République du Benin

Akokponhoué Houngnigbo Bertrand, PhD

Laboratoire d'Hydrologie Appliqué, Institut National de l'Eau (INE),

Université d'Abomey-Calavi, Cotonou, République du Benin

Youan Ta Marc, MC

Département des Sciences et Techniques de l'Eau et du Génie de

l'Environnement, U.F.R des Sciences de la Terre et des Ressources Minières

(STRM), Université de Cocody, Côte d'Ivoire

Agbahoungba George, PhD

Chaire Internationale en Physique Mathématique et Applications (CIPMA Chaire UNESCO), Université d'Abomey-Calavi, Cotonou,

République du Benin

\section{Abstract}

The hilly region is famous in Benin for its water supply problems because it is located in an area composed only of crystalline rocks. It is experiencing acute difficulties of access to water resources. The present study aims to map fracture networks from satellite images and to use high resolution electrical resistivity tomography for their ground locations. The methodological approach used consists of the use of remote sensing techniques for the structural mapping of lineaments and the application of electrical resistivity tomography for the validation of hydraulically active lineaments. The use of the Selective Principal Component Analysis techniques and Sobel (7x7) directional spatial filtering techniques made it possible to map the major lineaments. The validation of lineaments was made during several geophysical prospecting surveys of electrical resistivity tomography. The map 
generated as a result of satellite image processing has 638 linear structures ranging in size from 7 to $34 \mathrm{~km}$ and having two preferred directions $\mathrm{N0}{ }^{\circ}$ $\mathrm{N} 10^{\circ}$ and $\mathrm{N} 80^{\circ}-\mathrm{N} 90^{\circ}$. At the end of the geophysics, 13 lineaments were validated in major fractures with widths of the fissured-altered zone ranging from 15 to $40 \mathrm{~m}$ and whose depths are beyond $50 \mathrm{~m}$. The range of electrical resistivity characterizing these fractures varies from 100 to $800(\Omega . \mathrm{m})$.

Keywords: Lineaments, Electrical Resistivity Tomography, Crystalline Rocks, Water resources, Benin

\section{Introduction}

Avoir l'eau en quantité et en qualité à portée de main est, avec la nourriture, un des plus urgents besoins de l'homme sur terre. La rareté de l'eau engendre de nombreuses tragédies au sein des communautés. Au Bénin, et dans la région du Centre-Ouest, située en zone de socle cristallin, il arrive qu'on manque d'eau dans les ménages pour couvrir les besoins quotidiens. Cela est dû au taux élevé de forages négatifs de plus de $40 \%$ et le pourcentage de forages de débit inférieur à $1 \mathrm{~m}^{3} / \mathrm{h}$ avoisine les $85 \%$. Une des solutions à ces nombreux problèmes se trouve dans une meilleure connaissance des aquifères de fractures. En effet, les travaux de Lasm (2000) ont montré qu'une connaissance plus précise des aquifères fracturés est indispensable pour une meilleure localisation, exploitation et gestion durable de leurs ressources. L'une des approches fondamentales de nos jours dans l'étude de la fracturation est la télédétection (Akokponhoué et al, 2018). La télédétection constitue un bon outil dans l'étude des discontinuités tectoniques même les plus discrètes pour la prospection hydrogéologique et la recherche des eaux souterraines en milieux fracturés (Youan Ta et al, 2014 ; Jofack Sokeng, 2016 ; Galanos et al, 2006 ; Jourda et al, 2006). Cependant les résultats issus de l'utilisation des techniques de la télédétection sont confrontés aux problèmes de leurs fiabilités et de leurs validations (Lasm, 2000, Jourda, 2005, Youan Ta 2008). La télédétection spatiale fournie des informations de manière globale, rapide, quantitatives et homogènes (Jofack Sokeng, 2016). Ceci souligne l'obligation de procéder à la validation et au contrôle au sol des résultats issus de l'interprétation des images satellitaires. En effet, la géophysique en général et l'imagerie de tomographie de résistivité électrique en particulier, vient en appuie aux approches de la télédétection. Elle permet de déterminer avec une meilleure précision la position des discontinuités géologiques et de localiser les zones à fort intérêt hydrogéologique. L'utilisation d'une approche couplée télédétection et géophysique peut remédier au manque de données et réduire considérablement les incertitudes dans l'implantation des forages en zone de socle (Alle et al, 2018, Soro et al, 2017, Akokponhoué et al, 2018). Le présent travail, vise donc à cartographier et localiser avec précision les aquifères 
fracturés en vue d'une meilleure planification de l'approvisionnement des populations du Centre-Ouest Bénin en eau potable.

\section{Présentation de la zone d'étude}

La zone d'étude est située au Sud-Ouest du département des collines. Elle est localisée entre $7^{\circ} 35$ et $8^{\circ} 13$ Nord d'une part et $1^{\circ} 30$ et $2^{\circ} 60$ Est d'autre part. La zone d'étude partage ses frontières avec les communes de DassaZoumè et de Glazoué à l'Est, de Djidja au Sud, de Bantè au Nord et la République sœur du Togo à l'Ouest sur environ $65 \mathrm{~km}$ comme l'indique la figure 1. Elle s'étend sur près de $58 \mathrm{~km}$ de l'Ouest à l'Est et couvre une superficie de $2674 \mathrm{~km}^{2}$, soit $2,37 \%$ du territoire national avec une population totale de 144814 habitants (INSAE, 2016).

Sur le plan géomorphologique, on observe une pénéplaine rocheuse très accidentée avec des dômes isolés d'inselbergs et de petites chaînes dont la plus longue s'étend sur près de $20 \mathrm{~km}$ et de pittoresques collines. Le relief culmine entre 120 et $500 \mathrm{~m}$ avec des pentes variant entre 3 et $10 \%$ dans les sites agglomérés.
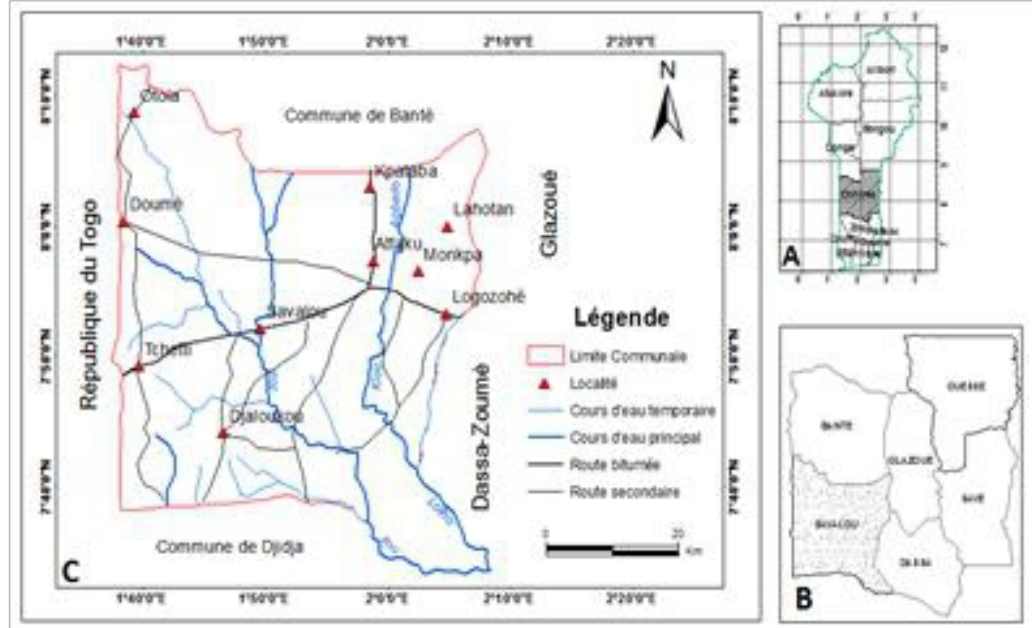

Figure 1: Carte de la zone d'étude

Il détermine en grande partie l'aptitude au ruissellement des terrains, l'infiltration et l'évaporation. Il s'agit d'une plaine érodée développée sur du gneiss et laissant en relief les éléments granitiques ou des carapaces ferrugineuses les plus résistantes. 


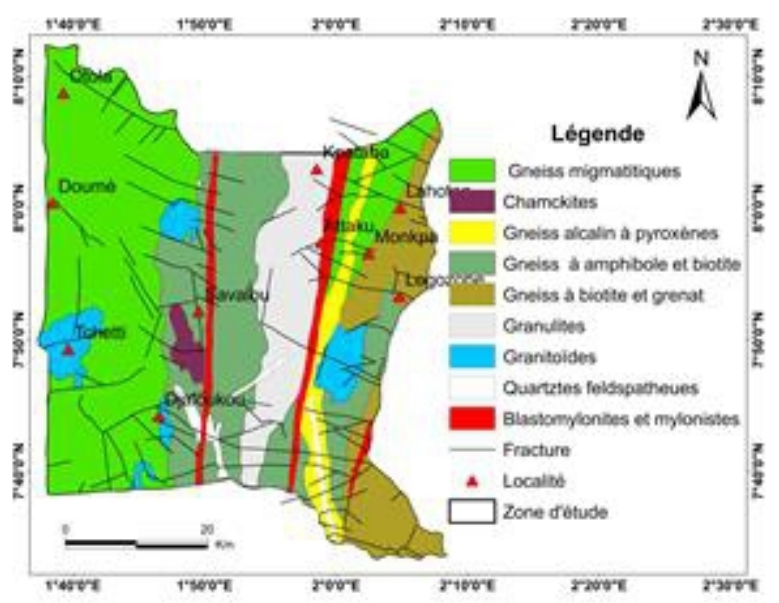

Figure 2: Carte géologique de la zone d'étude ((Breda, 1989), modifiée).

Sur le plan géologique, la zone se trouve sur un sous-sol composé de roches cristallines à faible altération ( $8 \mathrm{~m}$ en moyenne) et repose sur du matériel précambrien du vieux socle granito-gneissique. Les travaux de (Affaton, 1987) et de (Boukari, 1982 ; Adissin Glodji, 2012) ont montré la complexité géologique de cette zone d'étude. Il s'agit en effet, d'un ensemble lithologique et structural ayant subi plusieurs phases de déformation, de métamorphisme et de magmatisme. On retrouve ainsi les granites à biotite et/ou à porphyrique à Tchètti, les gneiss à biotite et/ou à amphibole de Dagadoho, les gneiss à biotite et hyperthème granulite de Monkpa et les blastomylonites et mylonites liés à l'accident de Kandi. Sur la figure 2, ces différents phénomènes géologiques sont matérialisés par de nombreuses fractures généralement structurées E-W et N-S.

$\mathrm{Au}$ plan hydrogéologique, la zone est caractérisée par une coexistence de deux réservoirs superposés. Il s'agit des réservoirs d'altérites et des réservoirs de fractures encore appelés aquifères discontinus. Alors que les réservoirs d'altérites ont une porosité primaire et un rôle capacitif du fait de leur nature argilo-sableuse, les réservoirs de fractures sont sous-jacents avec une porosité secondaire jouant un rôle transmissif. Les résultats de nombreux forages réalisés par les projets d'Hydraulique Villageoise dans le secteur montrent que le réservoir d'altérite présente une épaisseur très faible comprise entre 2 et $8 \mathrm{~m}$ avec des profondeurs de forage variant entre 38 et $80 \mathrm{~m}$, pour des débits de $0.7 \mathrm{à} 10 \mathrm{~m}^{3} / \mathrm{h}$. Le taux de réussite pour la réalisation des forages est de $30 \%$ à $70 \%$ pour l'hydraulique villageoise et de $20 \%$ à $30 \%$ pour l'hydraulique urbaine. 


\section{Matériel et méthodes}

\section{Données et matériel pour la cartographie des fractures}

Cette étude a nécessité l'utilisation d'image de Landsat 8 OLI de résolution 30x30 d'une scène (192-055). Cette image satellitaire a été téléchargée le 19 février 2017 sur le site de la NASA https://landsart.usgs.gov. Des fonds de carte topographique et géologique, toutes établient à l'échelle 1/200 000 par l'Institut Géographique National du Bénin en 1965 ont été utilisés. Ces données ont été traitées à l'aide des logiciels tel que ArcGis 10.3 et Envi 5.1. Après l'extraction des linéaments, les différentes familles de fractures (en nombres et en longueurs cumulées) ont été traitées avec le logiciel Rock Works 16.

\section{Données et matériel pour la validation linéamentaire}

Les données utilisées dans cette étude sont constituées de 12 panneaux de TRE (Tomographie de Résistivité Electrique) réalisés sur des linéaments majeurs identifiés après l'application des techniques d'extraction de ceux-ci par la télédétection. Les travaux de tomographie de résistivité électrique ont été faits dans les mois de Mai, Juin et juillet 2018 et dans cinq zones après le traitement des images. Le matériel de prospection de TRE pour la collecte des données sur le terrain, est composé d'un Syscal R2 plus Swicth 48 de la société Iris Instruments, avec ses accessoires (des bobines de raccordement ou flûtes, des électrodes, un hectomètre, des masses, une boussole, un GPS, des pinces crocodiles, un convertisseur de tension électrique DC/DC, une batterie $12 \mathrm{~V}$ etc.). Le traitement des données géophysiques a été effectué par les logiciels fournis avec l'appareillage tels que le Prosys II et DC2DInvRes.

\section{Méthodologie}

L'approche méthodologique utilisée dans cette étude se résume en 2 étapes. D'abord la méthode de télédétection appliquée aux images OLI pour la cartographie structurale. En suite, l'utilisation de la prospection géophysique par la TRE pour la localisation des fractures au sol.

\section{Approche de la télédétection pour la cartographie des linéaments}

La méthodologie à suivre pour l'identification du réseau de fractures dans la zone d'étude se résume au prétraitement des images satellitaires (correction radiométrique), à l'accentuation des discontinuités structurales et enfin à l'extraction des linéaments présents sur l'image. L'emploi de plusieurs méthodes de traitements numériques appliquées aux images OLI 8 à l'aide des techniques de fusion d'images (APCS) et des techniques de filtrages spatiaux directionnelles ont abouti à augmenter la perception de l'image en l'améliorant et en la rendant plus expressive et plus nette. 


\section{Prétraitement des images $\mathrm{OLI}$}

La phase de prétraitement des images satellitaires consiste à éliminer les bruits radiométriques dans les bandes, permettant de disposer d'une gamme très étendue d'images transformées et de faciliter leur l'interprétation. Les images OLI sont préalablement géo-référencées et corrigés géométriquement. Seules les corrections radiométriques ont été faites sur ces images. Cette correction a permis d'éliminer les bruits radiométriques dans les bandes OLI. La correction radiométrique s'est effectuée d'une part, par l'application de la transformée de Fourier (Smirnov, 1992) sur le canal panchromatique de OLI et la bande OLI 5 de Landsat et d'autre part, par l'utilisation de filtres passe bas (Moore et Waltz, 1983). La correction de l'effet atmosphérique et du relief a été faite pour réduire l'effet de brume sèche, assimilé à un facteur à caractère additif sur le signal radiométrique, de ces bandes, la technique proposée par ( Kouamé, 1999) a été adoptée dans la présente étude. Le ré-échantillonnage des pixels des bandes panchromatiques (OLI 8) a en outre été nécessaire pour ramener les pixels de $60 \mathrm{~m}$ à $30 \mathrm{~m}$ de côté. La méthode d'interpolation bilinéaire a été utilisée. Les images corrigées ont subi d'autres traitements afin d'améliorer leur perception visuelle en vue d'une meilleure cartographie des accidents géologiques de la région d'étude.

\section{Traitement des images OLI}

\section{Technique d'analyse en composante principale sélective}

Dans le souci de cartographier le maximum des discontinuités ayant affecté la zone d'étude, la technique de l'analyse en composantes principales sélectives (ACPS) a été appliquée. Elle a consisté à effectuer une première ACPS avec les bandes du visible 2, 3 et 4, ce qui a permis d'obtenir trois composantes $\mathrm{ACP} 1_{234}, \mathrm{ACP} 2_{234}$ et $\mathrm{ACP} 3_{234}$. Ensuite, une deuxième ACPS a été effectuée avec les bandes de l'infrarouge 6,7 et la bande panchromatique (OLI 8), ce qui a abouti également à l'obtention de trois autres composantes $\mathrm{ACP} 1_{678}, \mathrm{ACP} 2_{678}$ et ACP $3_{678}$. L'ensemble de ces composantes principales obtenues ont fait l'objet d'une interpretation visuelle dans un canal (noir et blanc). Cette technique a été inspirée de celle utilisée par Jourda, (2005), Youan Ta, (2008), Youan Ta et al, (2014) dans le socle précambrien de la Côte d'Ivoire et de Akokponhoué et al, (2017) sur une portion du segment NordOuest de la chaîne panafricaine des Dahoméyides au Bénin.

\section{Technique de filtrages spatiaux directionnels des linéaments}

Les filtres spatiaux représentent une autre méthode de traitement numérique utilisée pour le rehaussement d'une image. Ces filtres ont donné de très bons résultats lors de l'étude structurale du bassin versant de la Marahoué ( Biémi, 1992) des chaînes des Toura ( Kouamé, 1999) de la région de Korhogo ( Jourda, 2005) et de Bondoukou ( Youan Ta, 2008) en Côte d'Ivoire 
et avec (Akokponhoué et al, 2017) au Nord-Ouest du Bénin sur une portion de la chaîne panafricaine des Dahomeyides. Dans le cadre de cette étude, la technique du filtrage par fenêtre mobile $(1024 \times 1024)$ a été utilisée afin d'accentuer les discontinuités structurales au moyen des filtres spatiaux conçus à cet effet. Dans la présente étude, les filtres directionnels (type Sobel de taille 7x7) ont été appliquées sur les bandes OLI4, OLI6, ACP2 234 , ACP1678. Ces filtres directionnels sont présentés dans le tableau 1.

Tableau 1 : Filtres directionnels de type Sobel de taille $7 x 7$ affectés du poids $6:$ a) N-S ; b) E-W

\begin{tabular}{lllllll}
\hline 1 & 2 & 3 & 4 & 3 & 2 & 1 \\
\hline 2 & 3 & 4 & 5 & 4 & 3 & 2 \\
\hline 3 & 4 & 5 & 6 & 5 & 4 & 3 \\
\hline 0 & 0 & 0 & 0 & 0 & 0 & 0 \\
\hline-3 & -4 & -5 & -6 & -5 & -4 & -3 \\
\hline-2 & -3 & -4 & -5 & -4 & -3 & -2 \\
\hline-1 & -2 & -3 & -4 & -3 & -2 & -1
\end{tabular}

a) Filtre Sobel de direction N-S

\begin{tabular}{lllllll}
\hline 1 & 2 & 3 & 0 & -3 & -2 & -1 \\
\hline 2 & 3 & 4 & 0 & -4 & -3 & -2 \\
\hline 3 & 4 & 5 & 0 & -5 & -4 & -3 \\
\hline 4 & 5 & 6 & 0 & -6 & -5 & -4 \\
\hline 3 & 4 & 5 & 0 & -5 & -4 & -3 \\
\hline 2 & 3 & 4 & 0 & -4 & -3 & -3 \\
\hline 1 & 2 & 3 & 0 & -3 & -2 & -1
\end{tabular}

b) Filtre Sobel de direction E-W

\section{Technique de prospection géophysique.}

Pour l'investigation des travaux de terrain de la présente étude, le mode d'acquisition des données est programmé suivant la configuration dipôledipôle. En effet, les travaux de plusieurs auteurs (Baltassat et al, 2017), Roques, (2013), (Alle et al, 2018) ont montré que la configuration dipôledipôle (DD) offre une bonne résolution des terrains de sub-surface et des discontinuités verticales et horizontales. Les séquences d'acquisition des données sont programmées sous le logiciel Prosys II. Les investigations sur le terrain sont faites avec des profils de $240 \mathrm{~m}$ pour une distance inter-électrode de $5 \mathrm{~m}$ soit 48 électrodes au total. Les 12 panneaux électriques sont mis en œuvre sur 5 sites. Ces panneaux ont tous les mêmes longueurs avec des orientations différentes tout en restant perpendiculaire aux linéaments. Les paramètres de mesure (nombre de répétitions (stacks), durée d'injection, erreur acceptable, mode de mesure) sont définis et programmés dans l'appareil de mesure. Les mesures des profils sont filtrées et traitées sous le logiciel X2ipi, puis inversées avec le logiciel Res2dInv tout en intégrant la topographie le long des profils. Pour une bonne comparaison des différents panneaux électriques, il a été choisi et appliqué une échelle de couleur pour les différentes gammes de résistivités (compris entre 5 et 4000 Ohm.m) à l'ensemble de l'imagerie du sous-sol dans la zone d'étude. La figure 3 présente les zones de prospections et l'orientation des profils TRE. 


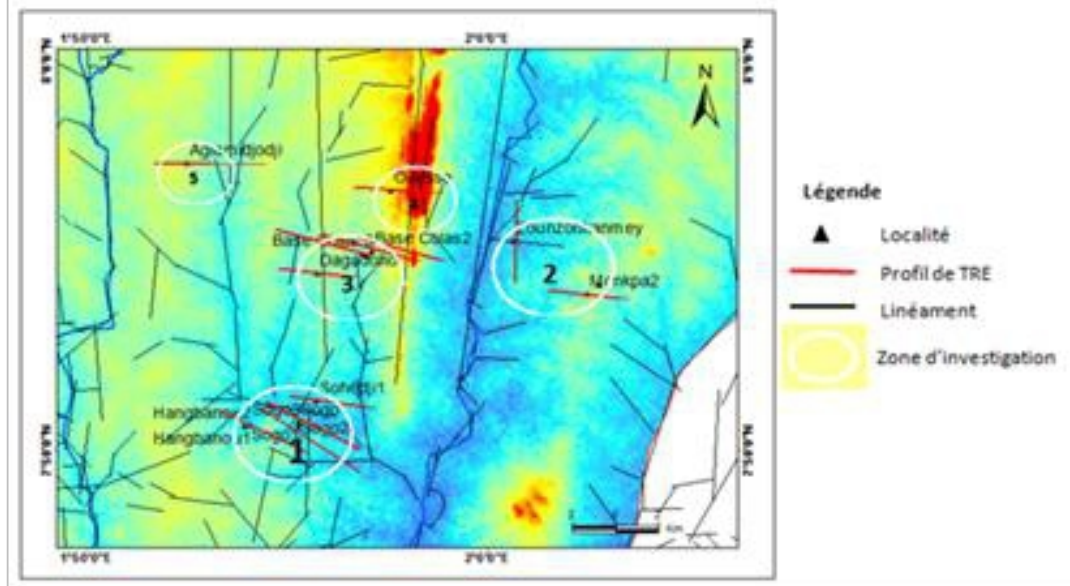

Figure 3:Présentation des zones de prospections et l'orientation des profils TRE

Les travaux de terrain pour la validation des linéaments à l'aide de la TRE ont été exécutés dans les cinq (5) zones. Ces zones sont choisies en fonction des formations géologiques, de la densité des linéaments, de la proximité des localités ou des cours d'eau. Elles sont numérotées de 1 à 5 sans aucune priorité. Ainsi, 13 linéaments majeurs ont été identifiés sur la carte des linéaments et ont fait objet de prospection géophysique.

\section{Résultats}

\section{Cartographie des linéaments régionaux à l'aide de la télédétection}

L'utilisation des filtres de Sobel de dimension 7 x7 sur les ACPS 234 (figure 4) et les ACPS 678 (figure 5) ont permis de mettre en évidence de nombreux linéaments structuraux sités au Centre-Ouest du Bénin. La figure 6 présente l'ensemble des linéaments majeurs issus des interpretations des images dérivées des différentes techniques de traitements. Ces linéaments, au nombre de 638 , ont des tailles et des directions variables pouvant atteindre plusieurs kilomètres. L'analyse de la figure 6 montre que $51 \%$ des linéaments ont une taille comprise entre 4 et $12 \mathrm{~km}, 35 \%$ des linéaments ont une taille comprise entre 12 et $25 \mathrm{~km}$ et environ $14 \%$ des linéaments dépassent $30 \mathrm{~km}$ de longueur. Cette étude a permis de cartographier plusieurs discontinuités géologiques dont les principales sont :

Le linéament allant d'Attakè à Kpataba (L11 AT-KB) de direction N10

Le linéament de Mokpa (L1MP) de direction $\mathrm{N}^{\circ}{ }^{\circ}$;

Le linéament de Savalou (L7SO) de direction $\mathrm{N}^{\circ}$ 


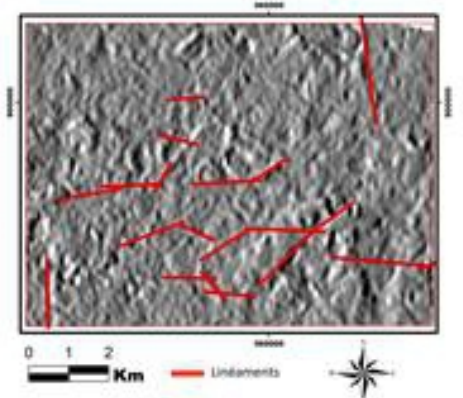

Figure 4: Sobel de direction N- S appliqué à Figure 5: Sobel de direction E-O appliqué à 1'ACPS 234 rehaussant les linéaments E-O

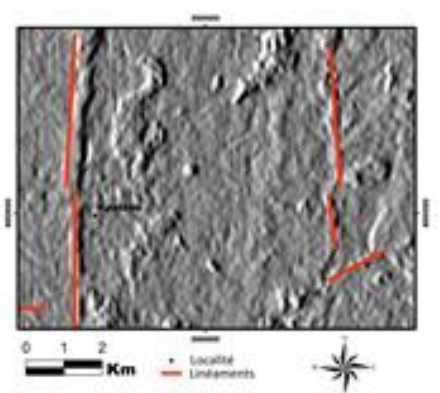

1'ACPS 678 rehaussant les linéaments N-S

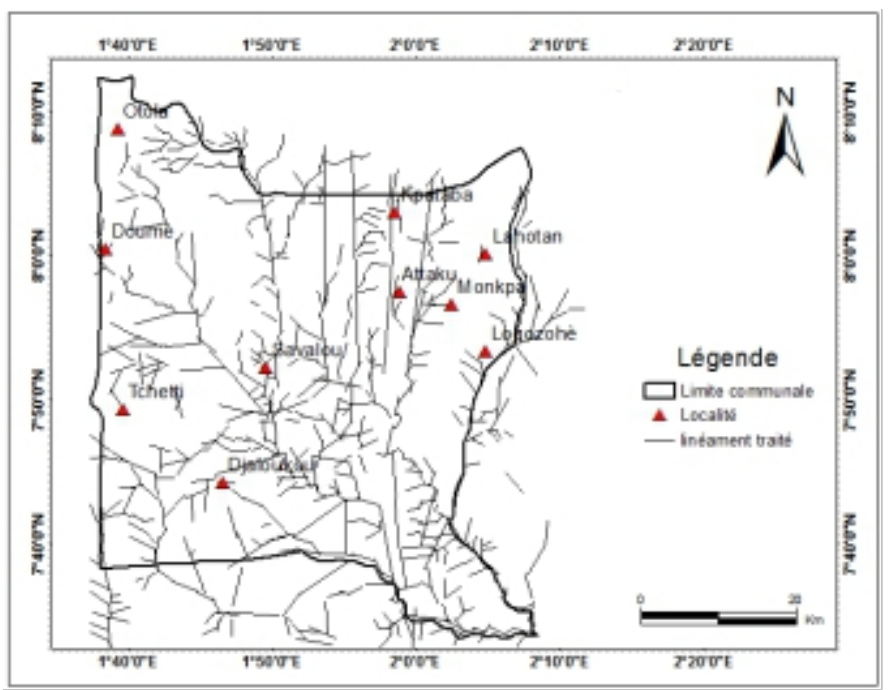

Figure 6: Carte des linéaments majeurs extraits de la zone d'étude

Le linéament de Tchètti (L12TT) de direction N70 ;

Le linéament de Doumè (L8DM) de direction N80 ;

Le linéament de Sogo (L6SG) de direction N10 ;

Le linéament de Sohèdji (L3SJ) de direction N0 ;

Le linéament allant de la base colas à Dagadoho (L4BC-DH) de direction $\mathrm{N} 10^{\circ}$.

Les 638 linéaments majeurs sont regroupés en 18 classes selon leur orientation suivant des croissances angulaires de 10 degrés sur une rosace directionnelle. L'analyse directionnelle de la fracturation du Sud-Ouest du Département des Collines en nombres et en longueurs cumulées (Figures 7a et $7 \mathrm{~b}$ ) montre une certaine homogénéité de la fracturation.

Les fractures les plus abondantes (N-S et E-W) sont aussi les plus longues. Les classes dominantes de la fracturation en nombres (Figure 7a) mettent en évidence deux directions prépondérantes, il s'agit respectivement de la 
direction $\mathrm{N}^{\circ}-10^{\circ}(\mathrm{N}-\mathrm{S})$, largement dominante, représentant plus de $6 \%$ en fréquences des fractures en nombre et la direction $\mathrm{N} 80^{\circ}-90^{\circ}(\mathrm{E}-\mathrm{W})$, dominante, représentant plus $4 \%$ en fréquences de fractures en nombre. Les autres classes dont les fréquences sont inférieures à $4 \%$ sont considérées comme minoritaires. Concernant la distribution de longueurs cumulées aucune classe de fracture ne dépasse les $10 \%$. La répartition des fractures au CentreOuest de Benin peut être donc qualifiée d'homogène. Cependant, deux classes de direction se distinguent de l'ensemble.
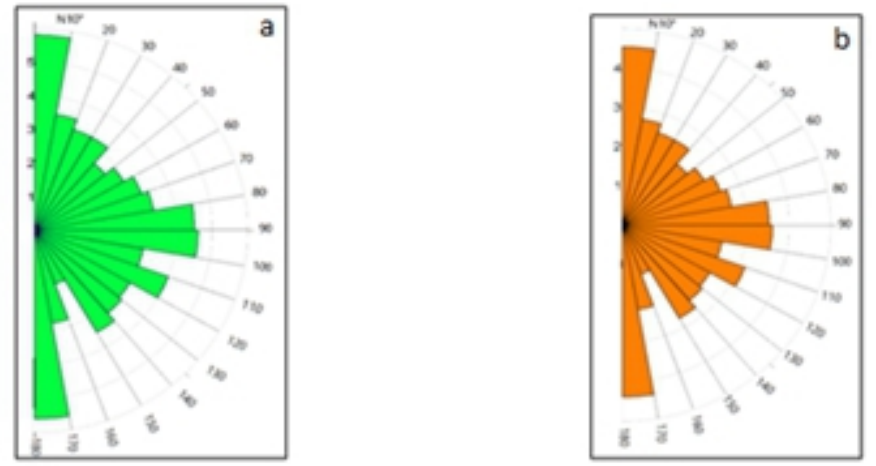

Figure 7: Rosaces directionnelles des linéaments au Sud-ouest du Département des Collines au Bénin: $a$-Fréquence en nombre de linéaments ; $b$-Fréquence en longueurs cumulées

Il s'agit des directions $\mathrm{N}^{\circ}{ }^{\circ} 10^{\circ}(\mathrm{N}-\mathrm{S})$, avec des fréquences en longueurs cumulées avoisinant les $5 \%$ et les directions $\mathrm{N} 80^{\circ}-90^{\circ}(\mathrm{E}-\mathrm{W})$ avec des fréquences en longueurs cumulées avoisinant les $4 \%$. A ces directions l'on peut ajouter les directions secondaires $\mathrm{N} 160^{\circ}-170^{\circ}$ (NE-SW) et $\mathrm{N} 90^{\circ}$ $100^{\circ}(\mathrm{NW}-\mathrm{SE})$ avec une fréquence inférieure à $4 \%$ et $3 \%$ en pourcentage de longueurs cumulées (Figure 7b). A cette étape, nous ne sommes pas en mesure d'affirmer que le réseau de linéament traité est une représentation fidèle de la réalité. Toutefois, nous pensons qu'il est fiable et très représentatif des mouvements tectoniques et des accidents géologiques dans la zone. Le réseau de linéaments est considéré comme étant le réseau de fracture de la zone d'étude. Ainsi, cette carte de linéaments est d'une importance capitale du fait qu'elle servira de base dans l'orientation de la prospection géophysique en vue d'identifier et de la validation des fractures les plus productives pouvant donner de forages à gros débits.

\section{Caractérisation géophysique}

Pour la présente étude, seuls les résultats de 4 panneaux sont présentés et interprétés. Cependant, le tableau 2 présente la synthèse des résultats des 12 panneaux après les travaux de prospection géophysique. 
Site de Sohedji dans la zone 1

L'imagerie de résistivité électrique du sous-sol de la figure 8 est obtenue sur

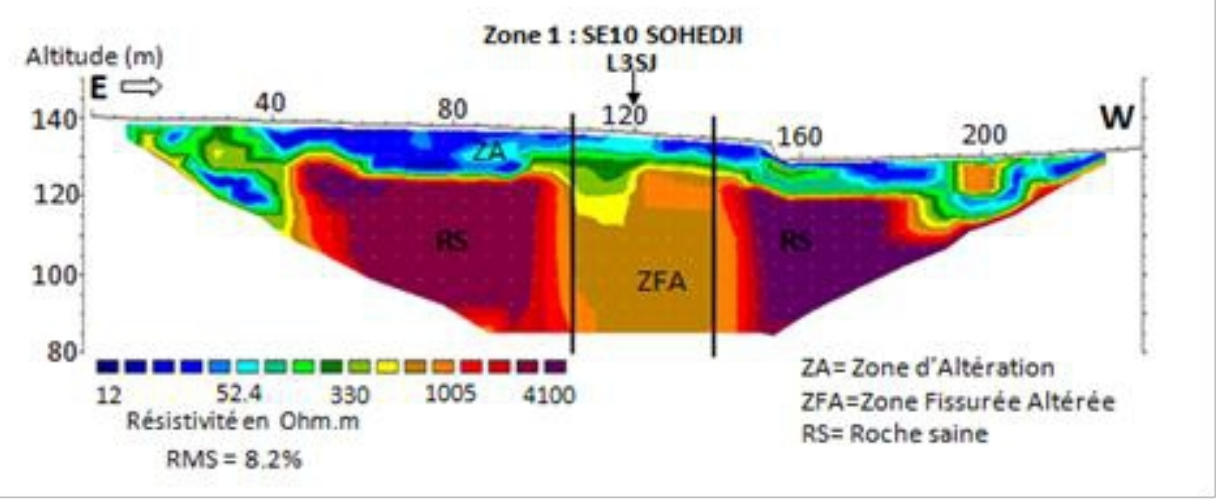

Figure 8: Panneau de TRE SE10 : Site de Sohèdji (zone 1)

le site de Sohèdji zone 1 . Le profil est réalisé perpendiculairement au linéament L3SJ. Le panneau de résistivité électrique présente des gammes de résistivité variant de 12 à $4200 \Omega$.m. Cette gamme de résistivité peut être répartie en 3 sous-gammes correspondant à différentes couches géologiques importantes du sous-sol. En effet, l'observation du panneau de résistivité électrique montre une première couche (1) horizontale de $8 \mathrm{~m}$ d'épaisseur le long du profil orienté E-W et conductrice avec de faibles résistivités allant de 12 à $70 \Omega$.m correspondante au gamme I. Sous la première couche superficielle, l'imagerie électrique du sous sol montre clairement une mince couche d'épaisseur variant entre 0.5 à $5 \mathrm{~m}$ tout le long du profil. Cette deuxième couche (2), peu résistante avec des résistivités de $350 \Omega . \mathrm{m}$ en moyenne peut atteindre $20 \mathrm{~m}$ de profondeur à l'Est, et à l'Ouest du profil.

Sous la deuxième structure, on a la troisième couche (3) constituée de blocs très résistants $(1500$ à $4000 \Omega$.m) et traversée par un couloir moins résistant (450 à $800 \Omega$.m) entre les abscisses 105 et $138 \mathrm{~m}$ au droit du linéament L3SJ qu'est le centre des mesures. L'épaisseur de cette dernière couche n'est pas connue $(>50 \mathrm{~m})$ pour défaut de profondeur d'investigation. Ainsi, le linéament L3SJ vu en surface par la télédétection est vu également en profondeur par TRE avec $32 \mathrm{~m}$ de largeur.

\section{Site de Monkpa dans la zone 2}

L'imagerie électrique (figure 9) du site de Monkpa zone 2 coupe les linéaments L1MP et L2MP. Elle présente des gammes de résistivités variant de 12 à $4300 \Omega$.m. Cette gamme de résistivité peut être répartie en 3 sousgammes correspondant aux différentes couches géologiques. En effet, on a une première couche (1) très conductrice superficielle de $10 \mathrm{~m}$ d'épaisseur tout le long du profil orienté E-W avec des résistivités faibles allant de 12 à $70 \Omega$.m. 


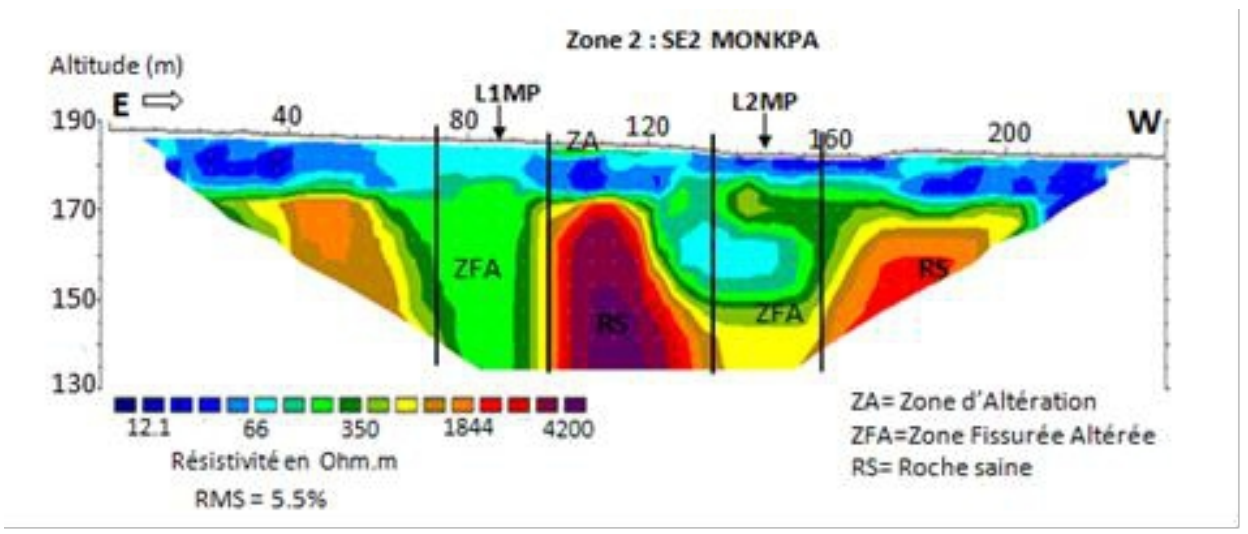

Figure 9:Panneau de TRE SE2 : Site de Monkpa (zone 2)

En dessous, on observe une deuxième couche (2) d'épaisseur variable pouvant dépasser $50 \mathrm{~m}$ localement à l'aplomb des linéaments (L1MP et L2MP) entre les abscisses 73 à $98 \mathrm{~m}$ et entre 135 à $160 \mathrm{~m}$ avec des résistivités moyennes allant de 100 à $1000 \Omega$.m. Enfin, la couche (3) très résistante avec des résistivités très élevées variant entre 1000 à $4300 \Omega$.m est très superficielle entre les abscisses 20 à 73, 98 à 134 et 160 à $200 \mathrm{~m}$. Sur l'imagerie du profil réalisé à Monkpa, on observe au total 3 terrains (couches) tous provenant de la transformation à divers degrés de la roche mère. Les deux linéaments observés sur l'image satellitaire sont nettement visibles sur le panneau électrique réalisé.

\section{Site de Dagadoho dans la zone 3}

L'imagerie électrique (figure 10), réalisée sur le site de Dagadoho zone 3 et sécant au linéament L4DH, présente des gammes de résistivités variant de 10 à $4000 \Omega$.m. Cette gamme de résistivité peut être regroupée en 3 sous- gammes représentant différentes couches géologiques. La première couche (1) superficielle de $12 \mathrm{~m}$ d'épaisseur est très conductrice tout le long du profil orienté E-W. Elle a des résistivités très faible allant de 12 à $150 \Omega . \mathrm{m}$ correspondant au gamme I. En dessous de cette couche 1, on observe une mince couche horizontale d'épaisseur faible 2 à $5 \mathrm{~m}$ avec des résistivités allant de 150 à $400 \Omega$.m. Au niveau de la couche (3), on observe une structure très résistante avoisinant $4000 \Omega$.m dont l'épaisseur est à l'infinie. La troisième e la deuxième couche sont traversées entre les abscisses 130 et $155 \mathrm{~m}$ par un couloir de même résistivité que la première couche. 


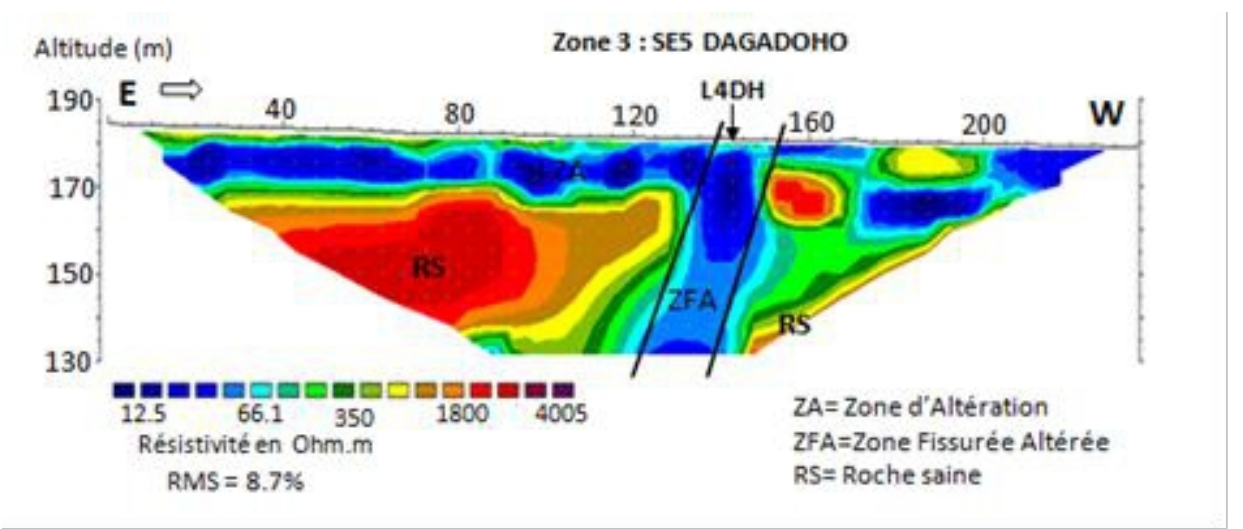

Figure 10: Panneau de TRE SE5: Site de Dagadoho (zone 3)

Le substratum de ce couloir est invisible sụr l'image de la figure 10. Ce couloir large de $\mathbf{1 5}$ m en trioyenne est la manirestation du linéament L4DH dans le sous-sol.

\section{Site de Aglamidjodji dans la zone 5}

L'image de la figure 11 réalisée sur le site de Aglamidjodji dans la zone 5 sécant au linéament L5AJ présente des gammes de résistivités allant de 10 à $4000 \Omega$.m. Cette gamme de résistivité peut être regroupée en 3 sousgammes représentant différentes couches géologiques. La première couche (1) superficielle avec une épaisseur variant de 5 à $15 \mathrm{~m}$. L'épaisseur de cette couche varie le long du profil orienté $\mathrm{E}-\mathrm{W}$ et peut atteindre $20 \mathrm{~m}$ par endroit. Elle est très conductrice avec des résistivités inférieures à $100 \Omega$.m (gamme I). Sous la première, on observe une deuxième (couche 2) horizontale qui se distingue par une résistivité électrique allant de 100 à $800 \Omega$.m (gamme II) avec une épaisseur variable entre 2 et $10 \mathrm{~m}$ le long du profil.

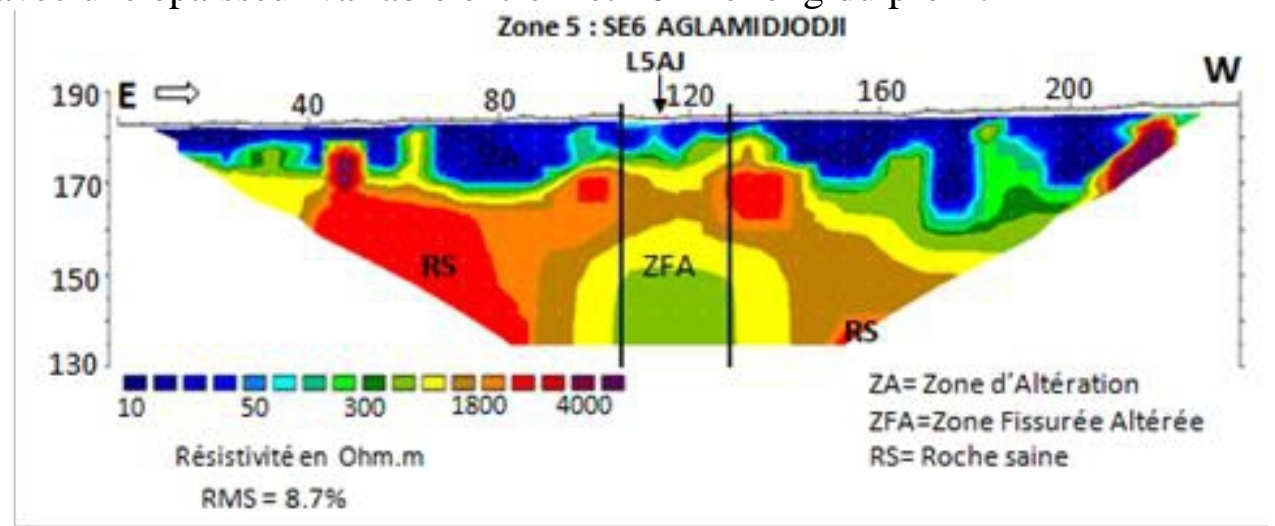

Figure 11: Panneau de TRE SE2 : Site de Aglamidjodji (zone 5)

La troisième couche toujours horizontale, a des résistivités allant de 1800 à $4000 \Omega$.m (gamme III) telle indiquée sur la figure 11. Cette troisième 
couche est traversée par un couloir de résistivité identique à celle de la deuxième couche. En effet, le linéament recherché (L5AJ) est baucoup plus visible sur ce panneau de résistivité électrique qu'à paritr de $20 \mathrm{~m}$ de profondeur. Cela est dû à une forte altération de la couche 3 à cette profondeur avec une largeur de 24 m environ. Le tableau 2 présente les caractéristiques des linéaments prospectés lors de la campagne de TRE pour leurs validations en fractures. Au regard des informations contenues dans ce tableau, nous constatons que l'orientation, le pendage, les gammes de résistivités, la largeur sans oublier l'épaisseur du recouvrement d'altérites au droit des différentes fractures varient suivant les sites investigués. Tous les liéaments identifés dans les 5 zones ont été validés par la tomographie de résistivité électrique. L'analyse du tableau indique que les linéaments cartographiés sont tous des structures géologiques linéaires avec des caractéristiques bien visibles en 2D. Ainsi, pour ces structures on a deux directions principales que sont le NordSud $(\mathrm{N}-\mathrm{S})$ et le l'Est - Ouest $(\mathrm{E}-\mathrm{W})$ avec des pendages allant de 0 à $10^{\circ} \mathrm{E}$ pour la direction $\mathrm{N}-\mathrm{S}$ et des pendages de 70 à $90^{\circ} \mathrm{S}$ pour les directions E-W. L'ouverture (largeur) des fractures dans la zone d'étude varie de 15 à $40 \mathrm{~m}$. Il faut noter que toutes ces fractures sont surmontées d'une faible épaisseur d'altérite ( $7 \mathrm{~m}$ en moyenne) provenant des produits d'altération soit du gneiss à grenat, soit du gneiss à biotite ou encore du gneiss lité principales formations géologiques de la zone. La gamme de résistivité traduisant la présence de structures géologiques linéaires en surface et correspondant aux couloirs de fractures dans le sous-sol varie de 100 à $800 \Omega$.m. Ces couloirs de fractures ont tous des profondeurs de plus de $50 \mathrm{~m}$, limite de la profondeur d'investigation de l'équipement utilisé (TRE avec 48 électrodes).

Tableau 2: Synthèse des résultats des panneaux de résistivité électrique au sol sur les 13 linéaments majeurs validés.

\begin{tabular}{|c|c|c|c|c|c|c|c|}
\hline \multirow{2}{*}{ Linéament } & \multicolumn{2}{|c|}{ Coordonnées } & \multirow[t]{2}{*}{$\overline{\mathbf{O}}$} & \multirow{2}{*}{$\begin{array}{l}P \quad \text { (en } \\
\text { degré) }\end{array}$} & \multirow{2}{*}{$\begin{array}{l}\text { Gr } \\
(\Omega . ~ m)\end{array}$} & \multirow{2}{*}{$\begin{array}{l}\mathbf{L} \\
(\mathbf{m})\end{array}$} & \multirow{2}{*}{$\begin{array}{l}\text { EA } \\
\text { (m) }\end{array}$} \\
\hline & long & lat & & & & & \\
\hline L13BA 1 & 1,94973 & 7,92037 & E-W & $90 \mathrm{~S}$ & 100 à 800 & 40 & 10 \\
\hline L17BA 2 & 1,94916 & 7,92043 & E-W & $70 \mathrm{~S}$ & 100 à 750 & 25 & 8 \\
\hline L34SG 1 & 1,92123 & 7,84531 & N-S & $10 \mathrm{E}$ & 450 à 800 & 32 & 5 \\
\hline L30SG2 & 1,92124 & 7,8453 & N-S & $7 \mathrm{E}$ & 250 à 600 & 25 & 7 \\
\hline L5AJ & 1,87464 & 7,95643 & $\mathrm{~N}-\mathrm{S}$ & $0 \mathrm{E}$ & 100 à 800 & 24 & 4 \\
\hline L3SJ & 1,93468 & 7,85005 & N-S & $0 \mathrm{E}$ & 250 à 800 & 30 & 6 \\
\hline L1MP1 & 2,04166 & 7,90053 & $\mathrm{~N}-\mathrm{S}$ & $0 \mathrm{E}$ & 250 à 800 & 25 & 5 \\
\hline L1MP2 & 2,04181 & 7,90048 & $\mathrm{~N}-\mathrm{S}$ & $0 \mathrm{E}$ & 150 à 800 & 25 & 5 \\
\hline L4DH & 1,92921 & 7,90945 & N-S & $10 \mathrm{E}$ & 100 à 800 & 15 & 4 \\
\hline L18OS & 1,95975 & 7,9446 & $\mathrm{~N}-\mathrm{S}$ & $5 \mathrm{E}$ & 100 à 500 & 35 & 10 \\
\hline L52ZE & 1,93978 & 7,90328 & E-W & $70 \mathrm{~S}$ & 150 à 750 & 38 & 8 \\
\hline L36HO1 & 1,90036 & 7,84567 & E-W & $80 \mathrm{~S}$ & 200 à 450 & 15 & 7 \\
\hline L21HO2 & 1,90969 & 7,84440 & $\mathrm{~N}-\mathrm{S}$ & $10 \mathrm{E}$ & 200 à 600 & 20 & 5 \\
\hline
\end{tabular}

Avec : $\mathrm{O}=$ Orientation; $\mathrm{P}=$ Pendage; $\mathrm{Gr}=$ Gamme de résistivité; $\mathrm{L}=$ Largeur et $\mathrm{EA}=$ Epaisseur de 1'Altérite 


\section{Discussion}

\section{Télédétection et cartographie structurale des linéaments}

Les différentes techniques de traitement spécifiques appliquées aux images Landsat OLI, à savoir les ACPS et techniques de filtrages spatiaux directionnelles des linéaments ont abouti à la réalisation de la carte des linéaments majeurs du Centre-Ouest de Bénin. Ces linéaments présentent deux directions majeurs à savoir : la direction $\mathrm{N}-\mathrm{S}$ et la direction $\mathrm{E}-\mathrm{W}$. Des résultats analogues ont été obtenus par Jourda, (2005), Youan Ta, (2008), et de Akokponhoué et al, (2018). Ces différents auteurs ont utilisé les techniques sus-citées dans l'extration des linéaments structuraux dans leurs zones d'étude respective et ont produit de très bons résultats. Les filtres directionnels utilisés dans la présente étude ont permis la mise en évidence de plusieurs structures linéaires dans la zone d'étude, c'est le cas par exemple des linéaments de Mokpa (L1MP) de Savalou (L7SO) de Tchètti (L12TT). Ces linéaments étaient jusque là mal connus car ils n'ont jamais été cartographiés au cours des études antérieures. Les linéaments cartographiés dans la présente étude de direction Nord-Sud, parallèles à l'allongement des structures géologiques, se sont mis en place lors de la phase de compression de l'orogenèse panafricaine. Ils sont de ce fait généralement colmatés. Par contre les linéaments transversales aux structures géologiques sont ouverts et donc plus productifs (Boussari, 1975). Pour (Boukari, 1982), ce sont plutôt les directions NE-SW et SE-NW qui soient les plus favorables aux fractures hydrauliquement actives et non les directions méridiennes ou subméridiennes. De même, (Engalenc, 1984) cité par le même auteur, la direction perpendiculaire (N-S à NNW-SSE) est également favorable pour la réalisation des forages à gros débit dans le secteur.

\section{Validation des linéaments par la tomographie de résistivité électrique}

De façon générale, l'imagerie au sol des 12 profils, montrent que la structuration géologique de la zone d'étude semble être la même. Ainsi, dans la zone d'étude on observe trois couches. En surface, on a une structure horizontale mince avec une épaisseur de $7 \mathrm{~m}$ en moyenne et très conductrice avec des résistivités inférieures à $150 \Omega$.m. Cette structure est la couche 1 correspondante à la zone altérée (ZA) de la roche constituée probablement d'un mélange de sable et d'argile. Sous cette première couche, on aperçoit un milieu souterrain constitué de structure géologique horizontale différenciée sur l'imagerie de la TRE par des résistivités moyennes comprise entre 150 et $800 \Omega$.m avec de faibles épaisseurs comprises entre 1 et $15 \mathrm{~m}$. Cette structure géologique (couche 2) retrouvée sur tous les panneaux électriques représente sans doute la zone fissurée altérée (ZFA). Cette zone fissurée peut être par endroit profonde dans la couche sous adjacente appelée couche 3 . La couche 3 telle que vue sur l'imagerie des différents panneaux électriques, avec des 
résistivités très élevées pouvant aller au-delà de $4000 \Omega$.m témoigne de la nature saine de la roche (RS). Toutefois, la roche saine est traversée par endroit par des couloirs de fractures en profondeur suite aux mouvements tectoniques. En effet, ce sont ces couloirs de fractures en profondeur qui sont matérialisés nettement par les linéaments majeurs en surface à l'issue du traitement des images satellitaires. Ces linéaments-fractures (couloirs de fractures ou discontinuités) sont reconnus sur l'imagerie par une zone de résistivité moyenne 150 à $800 \Omega$.m en profondeur dans du bloc très résistant avec des résistivités de $4000 \Omega$.m. Ainsi, les couloirs de fractures retrouvés sur toutes les sections de TRE réalisées dans la zone d'étude témoignent de l'effectivité de nombreuses fractures. Ces couloirs de fractures ont des ouvertures (largeurs) variant de 15 à $40 \mathrm{~m}$. La profondeur de ces couloirs de fractures (CF) ainsi que le toit de la roche saine (RS) ne sont pas connus dans cette étude par défaut de profondeur d'investigation de la TRE (profondeur maximale 50 $\mathrm{m})$. Ceci traduit la grande profondeur de la zone fracturée-altérée. Ces couloirs de fractures constituent les emplacements recherchés lors des campagnes de prospection géophysique pour l'implantation des futurs forages. Avec l'apport conjoint de la télédétection et de la géophysique (TRE), la localisation des couloirs de fractures devient plus aisée et plus sûre en zone de socle cristallin. Il est ainsi souhaitable que les praticiens de l'implantation de forage en milieu de socle s'approprient de cette méthodologie pour éviter des implantations hasardeuses avec ces conséquences au plan économique et social. Dans les milieux à double porosité/double perméabilité comme les zones de socle cristalline, la caractérisation de chaque portion est contraignante mais essentielle pour la mobilisation des eaux souterraines y afférentes. Pour (Roques, 2013) la description détaillée des structures aquifères dans ces zones par la géologie et par l'imagerie géophysique est un objectif incontournable pour la compréhension des réservoirs fracturés. Guidé par les résultats d'identification des structures linéaires majeures sur les images satellitaires de Landsat 8 puis les investigations géophysiques par la TRE, cela a permis d'identifier et de confirmer les discontinuités géologiques de la zone d'étude. Les linéaments extraits des images correspondent donc aux fractures sur le terrain. De nombreux travaux similaires de différents auteurs: (Savané, 1997; Kouamé, 1999), Jourda, et al, 2006, Descloitres et al, 2008, Soro et al, 2017, Alle et al, 2018) réalisés dans le même contexte ont abouti à la localisation précise des fractures issues des techniques de traitement spécifique de la télédétection dans les roches cristallines.

Les résultats obtenus à l'issu de ce travail offre un bon compromis en terme de localisation des fractures tectoniques sur le terrain entre la télédétection et la TRE. Contrairement aux travaux de Soro et al, (2017) à Sanon au Bukina Faso, la télédétection et la TRE ont permis de localiser et d'identifier les couloirs de fractures au Sud-ouest du département des collines au Bénin. 
Ainsi, avec l'imagerie de résistivité électrique du sous-sol, il serait possible d'implanter des forages dans les fractures qui seront plus productives Alle et al, (2018). La présente démarche est plus fiable pour augmenter la probabilité de succès lors des travaux d'implantation des forages en zone de socle cristallin. Connaissant les caractéristiques et la géométrie de la fracture, le forage sera idéalement implanté dans le lit de la fracture.

\section{Conclusion}

Le présent travail a pris en compte 2 activités à savoir, l'extraction des linéaments structuraux à partir du traitement des images satellitaires par les méthodes spécifiques de la télédétection et la localisation précise au sol de ces linéaments structuraux par la prospection géophysique notamment la TRE. Il ressort des travaux de la télédétection au Centre-Ouest du Bénin qu'un vaste réseau de linéaments majeurs existe. Ce réseau est composé de 638 linéaments majeurs en surface avec des directions dont les plus représentatives sont celles des classes de direction N-S (0-10), E-W (70-90).

Quant aux travaux d'investigations de la TRE, 12 profils de panneaux électriques ont été réalisés plus ou moins perpendiculairement aux linéaments. L'imagerie de résistivité électrique de ces panneaux a permi de valider 13 linéaments en fractures dans le sous-sol. Ces fractures ont des ouvertures de 15 à $40 \mathrm{~m}$ de large avec des profondeurs de plus de $50 \mathrm{~m}$. Ces fractures sont probablement de potentiels réservoirs d'eau souterraine dans cette zone qu'il faille à tout prix localiser pour une meilleure planification de l'approvisionnement des populations en eau potable. Nous comptons poursuivre les investigations par la réalisation de forages sur quelques zones identifiées dans cetteétude afin de comparer les résultats obtenus à ceux des travaux de forage.

\section{Remerciements}

Cette étude a été réalisée grâce au Projet «Mesure d'urgence pour le renforcement du système d'Alimentation en Eau Potable de la ville de Savalou» financé par L'Etat Béninois. Le contenu du présent papier relève de la seule responsabilité des auteurs qui, voudraient remercier et témoigner leur reconnaissance aux instructeurs anonymes pour leurs critiques, commentaires et suggestions qui ont contribué à l'amélioration du présent document. Les auteurs voudraient aussi remercier les responsables du Bureau d'Etudes CANAL-EAU Bénin qui ont accepté de nous accompagner pour les travaux de terrain.

\section{References:}

1. Adissin Glodji L. (2012). La zone de cisaillement de Kandi et le magmatisme associé dans la région de Savalou-Dassa (Bénin): étude 
structurale, pétrologique et géochronologique. Sciences de la Terre. Université Jean Monnet Saint-Etienne; Université d'Abomey-Calavi (Bénin), $277 \mathrm{p}$.

2. Affaton P. (1987). Le bassin des voltas (Afrique de l'ouest) : une marge passive du protérozoïque supérieur tectonisé au panafricain. Thèse d'état, volume 2, Université d'Aix-Marseille,449 p.

3. Akokponhoué, H. B., Yalo, N., Lasm, T., Youan Ta, M., Alassane, A., Kouamé K. J., Akokponhoué, Y. N., Hounton, C.C., Suanon F. (2017). Contribution of Remote Sensing to the Structural Mapping of Aquifers with Large Water Flows in the Crystalline Hard-Rock in the Department of Donga (North-West of Benin).Int. J. of Emerging Tech. and Adv. Eng., Vol. 7, 392-403 p.

4. Akokponhoué H. B., Yalo N., Adissin Glodji L., Alassane A., Agbahungba G., Lasm T. (2018). Cartographies structurale des accidents géologiques majeurs du socle cristallin du département de 1 a Donga (Nord-Ouest du Benin): Apport des images landsat-8 Oli et des données de terrain. Int. J. of Innovation and Applied Stu, Vol. 24 No. 3 Oct. 2018, pp. 1266-1283.

5. Alle C., Descloitres M., Vouillamoz J.M., Yalo N., Lawson F.M.A., Adihou A. C. (2018). Why 1D electrical resistivity techniques can result in inaccurate siting of boreholes in hard rock aquifers and why electrical resistivity tomography must be preferred: the example of Benin, West Afric, Journal of African Earth Sciences 139, 341-353 p.

6. Baltassat J-M, Maurice B., Reninger P-A (2017). Tomographies de résistivité électrique appliquée à l'implantation d forages AEP sur sites à Mayotte. Rapport final. BRGM/RP- 67169-FR, 71 p., 32ill, 4 tabl, 14 ann. Biemi J. (1992). Contribution à l'étude géologique, hydrogéologique et par télédétection des bassins versants subsahéliens du socle précambrien d'Afrique de l'Ouest : Hydrostructurale, hydrodynamique, hydrochimie et isotopie des aquifères discontinus de sillons et aires granitiques de la Haute Marahoué(Côte d'Ivoire).Thèse de Doctorat. ès Sc. Nat.Univ Abidjan, $493 \mathrm{p}$.

7. Boukari M. (1982). Contribution a l'étude hydrogéologique des régions de socle de l'Afrique intertropicale : l'hydrogéologie de la région de Dassa-zoumè (Benin). Thèse Université de Dakar, 137 p.

8. Boussari W.T. (1975). Contribution à l'étude géologique du socle cristallin de la zone mobile pan-africaine (région central du Dahomey). Thèse $3 e$ cycle, Univ. Besançon., $105 \mathrm{p}$.

9. Breda, 1989. Notice explicative de la carte géologique à $1 / 200000$. Feuilles Pira-Savè, Abomey-Zagnanado, Lokossa-Porto-Novo, 77p. 
10. Descloitres M., Ruiz L., Sekhar M., Legchenko A., Braun J.-J., Mohan kumar M. S., and Subramanian S. (2008). Characterization of seasonal local rechargeusing electrical resistivity tomography and magnetic resonance sounding. Hydrological Processes, 22(3), 384-394 p.

11. Engalenc M. (1984). Evaluation du programme hydraulique villageoise 2ème fed (enquête hydrogéologique). Technical report, Republique du Dahomey, Ministère des travaux publics,de la construction et de l'habitat.

12. Galanos I., Rokos D. (2006). A statistical approach in investigating the hydrogeological significance of remotely sensed lineaments in the crystalline mountainous terrain of the island of Naxos, Greece. Hydrogeology journal vol.14, pp 1569-1581.

13. INSAE ou Institut National de la Statistique et de l'Analyse Economique (2016). Effectifs de la population des villages et quartiers de ville du Bénin (RGPH-4, 2013). 83 p.

14. Jofack sokeng V.C. (2016). Cartographie des potentialités en eaux souterraines dans les Hauts Plateaux de l'Ouest-Cameroun: contribution de la Télédétection (optique et radar), des Systèmes d'Informations Géographiques et des Réseaux de Neurones, Thèse Université Félix Houphouet Boigny, 267 p.

15. Jourda J.P. (2005). Méthodologie d'application des techniques de télédétection et des systèmes d'information géographique à l'étude des aquifères fissurés d'Afrique de l'ouest. Concept de l'Hydrotechnique spatiale : cas des zones tests de la Côte d'Ivoire. Thèse de doctorat ès Sciences Naturelles, Université de Cocody-Abidjan, Côte d'Ivoire, $429 \mathrm{p}$.

16. Jourda J. P., Saley M. B., Djagoua E. V., Kouame K. J., Biemi J. et Razack M. (2006). Utilisation des données ETM+ de Landsat et d'un SIG pour l'évaluation du potentiel en eau souterraine dans le milieu fissuré précambrien de la région de de Korhogo (nord de la Côte d'Ivoire) : approche par analyse multicritère et test de validation. Revue de Télédétection, vol. 5, n 4, 339-357 p.

17. Kouame K.F. (1999). Hydrogéologie des aquifères discontinus de la région semi-montagneuse de Man-Danané Ouest de la Côte d'Ivoire). Apport des données des images satellitales et des méthodes statistique et fractale à l'élaboration d'un système d'information hydrogéologique à référence spatiale.Thèse de $3^{\mathrm{e}}$ cycle,Université de CocodyAbidjan,Côte d'Ivoire, $194 \mathrm{p}$.

18. Lasm T. (2000). Hydrogéologie des réservoirs fracturés de socle : Analyses statistiques et géostatistique de la fracturation et des propriétés hydrauliques. Application à la région des montagnes de 
Côte d'Ivoire Domaine archéen), Thèse de doctorat, Université de Poitiers, $272 \mathrm{p}$.

19. Moore G. K., Waltz F. A. (1983). Objective procedure for lineament enhancement and extraction: Photogrammetric Engineering and Remote Sensing, Vol. 49, n5, 641-647 p.

20. Roques C. (2013). Hydrogéologie des zones de faille du socle cristallin : Implications en termes de ressources en eau pour le Massif Armoricain. Thèse de doctorat Université de Rennes 285 p.

21. Savane I. (1997). Contribution à l'étude géologique et hydrogéologique des aquifères discontinus du socle cristallin d'Odienné (Nord-ouest de la Côte d'Ivoire).Apport de la télédétection et d'un système d'information hydrogéologique à référence spatiale. Thèse d'Etat,Université Cocody, 396p.

22. Smirnov M. X. (1992). Digital Filtering of lineament patterns: Sovietic Remote Sensing, $\mathrm{n}^{\circ} .1,101-111 \mathrm{p}$.

23. Soro D.D., Koita M., Biaou C.A., Outoumbe E., Vouillamoz J.-M., Yacouba H., Guerin R. (2017). Geophysical demonstration of the absence of correlation between lineaments and hydrogeologicallyusefull fractures: case study of the Sanon hard rock aquifer (central northern Burkina Faso). J. Afr. Earth Sci. 2-25 p.

24. Yesou H., Pion J. C., Besnus Y., et Saint Jean R. (1993). Amélioration des données SPOT pour la cartographie structurale en milieu tropical. Exemple de la région des chapeaux de fer de Pagala Togo). III èmes Journées Scientifiques du Réseau Télédétection UREF, Toulouse, 1316 novembre 1990, pp. 143-164. In:J.M. Dubois, F. Blascodir), Outil microinformatique et Télédétection de l'évolution des milieux, PUQ/AUPELF UREF.Ed, $492 \mathrm{p}$.

25. Youan Ta M. (2008). Contribution de la télédétection et des systèmes d'informations géographiques à la prospection hydrogéologique du socle précambrien d'Afrique de l'Ouest : Cas de la région de Bondoukou Nord Est de la Côte d'Ivoire. Thèse de doctorat, Université de Cocody-Abidjan (Côte d'Ivoire), $236 \mathrm{p}$.

26. Youan Ta M., Kouame K. F., Koudou A., Adja M. G., Baka D., Lasm T., Lasme, D. O., Jourda J. P., Biemi J. (2014). Apport de la Cartographie lithostructurale par imagerie satellitaire Landsat 7 à la connaissance des aquiferres du socle précambrien de la région de Bondoukou (Nord-Est de la Côte D'Ivoire). International Journal of Innovation and Applied Studies. Vol. 7 n .3 Aug. 2014, 892-910 p. 